\title{
Build Sustainable Cities!
}

\author{
Qian Yang \\ School of North China Electric Power University, Baoding 071000, China \\ 1026833458@qq.com
}

\begin{abstract}
Many communities are implementing smart growth initiatives. Smart growth is about helping every town and city become a more economically prosperous, more socially equitable, and more environmentally sustainable place to live in. This task is more important than ever because the world is rapidly urbanizing. Therefore, it is crucial to make it clear how to implement smart growth theories effectively into city design around the world. We develop a model for the task, with the major goal of measuring the success of smart growth of a city. To begin with, six factors are selected from the aspects of economy, social equity and environment. They are traffic saturation degree, average GDP, housing ownership rate, employment rate, average green area and density of building. Then we successfully finger out the weight vector of them by using AHP. Based on the ranking vector we designed by fuzzy composite operation, we can obtain the comprehensive evaluation value. In order to prove the accuracy of our evaluation system, the model testing is also made. Taking Bellevue as example, the result corresponds to the truth. It indicates that our evaluation system is useful and its results are convincing and realistic.
\end{abstract}


Keywords: smart growth, sustainable, factors, AHP.

\section{Introduction

$$
V=\left\{v_{1}, v_{2}, v_{3}, v_{4}\right\}
$$

The world is urbanizing rapidly nowadays, which results in more and more percentage of the world's population being added to the urban population. It is crucial to ensure cities to become more economically prosperous, socially equitable, and environmentally sustainable places to live in. The problem is to define a matrix to measure the success of the smart growth of a city, which makes sense to begin by designing a series of parameters to describe a city. Therefore, the quantitative calculation to cities becomes possible. Then, evaluation standard should be designed to make it clear whether a city is sustainable or not. Scientificity test is essential to prove that our evaluation system is reasonable and results are convincing and realistic.

\section{Model Building}

\subsection{Define Evaluation Factors Set}

Suppose $U=\left\{u_{1}, u_{2}, \ldots, u_{6}\right\}$ are the factors which could evaluate the level of sustainability of a city. Those factors are traffic saturation degree, average GDP, housing ownership rate, employment rate, average green area and density of building.

\subsection{Define Evaluation Rank Set}

Suppose are the ranks of the factors above. The number of the rank is equal to four.

Standard processing to evaluation indexes is based on the following formula: 


$$
W_{i j}=C_{i j} \int \sqrt{\frac{1}{n} \sum_{j=1}^{n}\left(C_{i j}-\overline{C_{i j}}\right)^{2}}(i=1,2, \ldots, m ; j=1,2, \ldots, n)
$$

The of the first and the fourth ranks have the unique maximum and minimum, while the others have a certain range. In this case, can be regarded as the average of its range.

\subsection{Establish Judgment Matrix}

We can obtain the membership degree $r_{i j}$ of each factor based on the membership function. Then, we acquire the single factor evaluation set for each factor: $r_{i j}=\left\{r_{i 1}, r_{i 2}, r_{i 3}, r_{i 4}\right\}$.

In vey of the seven factors, we can get a matrix for each city which is going to be evaluated. And this matrix is the fuzzy relations

$$
R=\left(r_{i j}\right)_{6 \times 4}=\left[\begin{array}{cccc}
r_{11} & r_{12} & r_{13} & r_{14} \\
\vdots & \vdots & \vdots & \vdots \\
r_{61} & r_{62} & r_{63} & r_{64}
\end{array}\right]
$$

Normalization processing to this matrix, then

\subsection{Calculate the Single Factor Weight}

We can obtain the weight matrix by using AHP arithmetic.

In this way, we get the weight vector of the six factors. (The program code is given in the appendix of this paper.)

$$
\Gamma r-1
$$

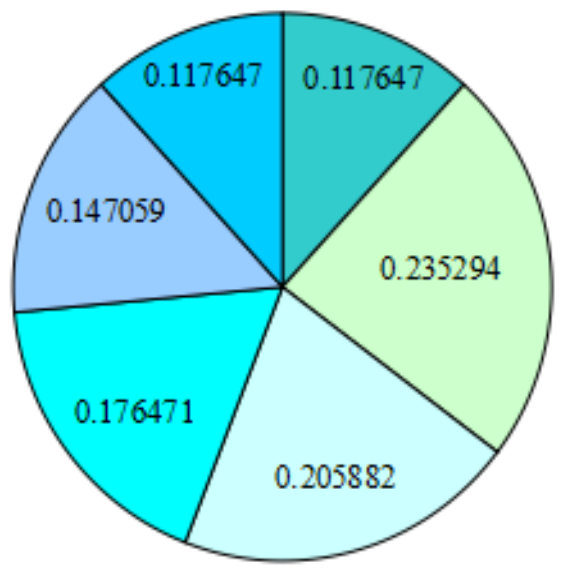

$\square$ traffic saturation degree

$\square$ aver age GDP

$\square$ housing ownership rate

Demployment rate

$\square$ aver age green area

$\square$ density of building 
Figure 1

\subsection{Fuzzy Composite Operation}

Membership vector can be calculated by using weight matrix A and fuzzy relationship matrix R.[1]

$$
B=\left(b_{1}, b_{2}, b_{3}, b_{4}\right)=A^{*} R=\left(a_{1}, a_{2}, a_{3}, a_{4}\right) *\left[\begin{array}{cccc}
r_{11} & r_{12} & r_{13} & r_{14} \\
r_{21} & r_{22} & r_{23} & r_{24} \\
\vdots & & & \vdots \\
r_{71} & r_{72} & \cdots & r_{74}
\end{array}\right]
$$

\subsection{Comprehensive Evaluation}

Design rank vector $C=(10,8,6,4)^{T}$, hence, we can get the final comprehensive evaluation result:

$$
P=B^{*} C
$$

$\mathrm{P}$ is a certain value for each city. It can reflect the comprehensive level of a city. The higher the $\mathrm{P}$ is, the more sustainable the city is. [2]

\section{Model Testing}

To ensure the scientificity of our evaluation procedure, we take Bellevue, Washington as example to test our evaluation system. [3] Based on the data we have got, calculation result is as shown below:

$$
\begin{gathered}
\mathrm{B}=(0.5247,0.4753,0,0) \\
\mathrm{P}=9.0494
\end{gathered}
$$

From this result, we come to the conclusion that this city is sustainable, which is accord with the fact. Afterwards, we have the reason to believe that our evaluation procedure is scientific and the evaluation result is convincing.

\section{Conclusion}

The factors which could describe the city are traffic saturation degree, average GDP, housing ownership rate, employment rate, average green area and density of building. It is reasonable that we believe these factors could describe the city adequately after testing. 
We conclude that the $\mathrm{P}$ value can reflect the comprehensive level of a city. The higher the $\mathrm{P}$ is, the more sustainable the city is.

\section{Acknowledgments}

This work was finish under the direction of my tutor, Ms.Liu. She helped me a lot in the grammar of the article. At the same time, thanks to the documents provided by CNKI and Google.

\section{References}

[1] Haifeng Ma. Study on the Intensive Utilization of Land Evaluation in Development. Zone Based on Smart Growth [D]. Chang'an University,2010.

[2] Juliang Jin, Yiming Wei, Jing Ding. Fuzzy comprehensive evaluation model based on improved analytic process [J]. Journal of Hydraulic Engineering, 2004, (03):65-70.

[3] https://www.data.gov/local/ 